Research Article

\title{
Partial Slip Flow and Heat Transfer over a Stretching Sheet in a Nanofluid
}

\author{
Rajesh Sharma, ${ }^{1}$ Anuar Ishak, ${ }^{1}$ and Ioan Pop ${ }^{2}$ \\ ${ }^{1}$ School of Mathematical Sciences, Faculty of Science and Technology, Universiti Kebangsaan Malaysia (UKM), \\ 43600 Bangi, Selangor, Malaysia \\ ${ }^{2}$ Department of Mathematics, Babeş-Bolyai University, 400084 Cluj-Napoca, Romania
}

Correspondence should be addressed to Anuar Ishak; anuarishak@yahoo.com

Received 2 February 2013; Revised 18 March 2013; Accepted 26 March 2013

Academic Editor: Jian Li

Copyright (c) 2013 Rajesh Sharma et al. This is an open access article distributed under the Creative Commons Attribution License, which permits unrestricted use, distribution, and reproduction in any medium, provided the original work is properly cited.

\begin{abstract}
The boundary layer flow and heat transfer of a nanofluid over a stretching sheet are numerically studied. Velocity slip is considered instead of no-slip condition at the boundary as is usually appears in the literature. The governing partial differential equations are transformed into ordinary ones using a similarity transformation, before being solved numerically. Numerical solutions of these equations are obtained using finite element method (FEM). The variations of velocity and temperature inside the boundary layer as well as the skin friction coefficient and the heat transfer rate at the surface for some values of the governing parameters, namely, the nanoparticle volume fraction and the slip parameter are presented graphically and discussed. Comparison with published results for the regular fluid is presented and it is found to be in excellent agreement.
\end{abstract}

\section{Introduction}

The fluid flow past a stretching plate was first investigated by Crane [1], where an exact analytical solution to the Navier-Stokes equations was reported. This problem was then extended to a permeable surface by P. S. Gupta and A. S. Gupta [2]. Grubka and Bobba [3] considered a more general case with power law surface temperature variation. They reported a series solution to the energy equation in terms of Kummer's functions and presented several closed-form analytical solutions for specific conditions. Further, the unsteady flow past a stretching sheet was investigated by Andersson et al. [4], Ali and Mehmood [5], Ishak et al. [6-8], Hayat et al. [9], Sharma [10], and Sharma et al. [11] among others. The study of flow and heat transfer past a stretching sheet is important due to its many industrial applications such as in the polymer industry, where one deals with a stretching plastic sheet. The quality of the final product depends on the rate of heat transfer at the stretching surface (Sparrow and Abraham [12]).

Nanofluid is a new class of fluid with nanosized particles dispersed in a poor thermal conductivity base fluid, such as water and ethylene glycol, to increase its thermal conductivity. These particles, generally metal or metal oxide, increase conduction and convection coefficient, allowing for more heat transfer out of the coolant (Choi and Eastman [13]). It seems that the term nanofluid was first introduced by Choi and Eastman [13] and it was adopted by many researchers. The materials with sizes of nanometers possess unique physical and chemical properties. They can flow smoothly through microchannels without clogging them because they are small enough to behave similarly to liquid molecules (Khanafer et al. [14]). They are also very stable and have no additional problems, such as sedimentation, erosion, additional pressure drop, and non-Newtonian behavior, due to the tiny size of nanoelements and the low volume fraction of nanoelements required for conductivity enhancement. A very interesting critical synthesis of the variants within the thermophysical properties of nanofluids has been recently presented by Khanafer and Vafai [15]. It has been shown that the experimental results for the effective thermal conductivity and viscosity reported by several authors are in disagreement. Thus, theoretical and experimental studies are essential to clarify the discrepancies in the results and in proper 
understanding of heat transfer enhancement characteristics of nanofluids. The study by Khanafer and Vafai [15] shows that it is not clear which analytical model should be used to describe the thermal conductivity of nanofluids. Additional theoretical and experimental research studies are required to clarify the mechanisms responsible for heat transfer enhancement in nanofluids. Correlations for effective thermal conductivity and viscosity are synthesized and developed in [15] in terms of pertinent physical parameters based on the reported experimental data. The broad range of current and future applications of nanofluids is discussed in the review article by Wong and Leon [16], which includes automotive, electronics, biomedical, and heat transfer applications besides other applications such as nanofluid detergent. In a recent review article by Saidur et al. [17], the authors also presented some applications of nanofluids in industrial, commercial, residential, and transportation sectors based on the available literatures. A critical review of the state-ofthe-art of nanofluids research for heat transfer application was conducted by Wen et al. [18], Mahian et al. [19], and so forth. The enhanced thermal behavior of nanofluids could provide a basis for an enormous innovation for heat transfer intensification, which is of major importance to a number of industrial sectors including transportation, power generation, micromanufacturing, thermal therapy for cancer treatment, chemical and metallurgical sectors, and heating, cooling, ventilation, and air-conditioning. Nanofluids are also important for the production of nanostructured materials for the engineering of complete fluids, as well as for cleaning oil from surfaces due to their excellent wetting and spreading behavior (Ding et al. [20]). The capability of nanofluids to enhance thermal conductivity has attracted the interest of fluid dynamics community to conduct further studies. As a result, the research on nanofluids has progressed rapidly. The number of research articles and citations found in Web of Science and SCOPUS under "nanofluids" shows exponential increase, indicating the high interest in research activities in this topic. In the present paper, we consider the flow and heat transfer over a stretching sheet immersed in a nanofluid, with velocity slip effect at the boundary. The nanofluid model proposed by Tiwari and Das [21], which analyzes the behavior of nanofluids taking into account the solid volume fraction, is employed in the present paper.

\section{Problem Formulation}

Consider a two-dimensional flow over a flat sheet with heat transfer in a water based nanofluid containing $\mathrm{Cu}, \mathrm{CuO}$, $\mathrm{Al}_{2} \mathrm{O}_{3}$, or $\mathrm{TiO}_{2}$ nanoparticles. We assume that the sheet coincides with the plane $y=0$ and the flow is confined to $y>0$. Two equal and opposite forces are applied along the $x$-axis so that the sheet is stretched keeping the origin fixed. It is assumed that the sheet is stretched with velocity $u_{w}=c x$, where $c>0$ is the stretching rate. It is also assumed that the base fluid (i.e., water) and the nanoparticles are in thermal equilibrium. The thermophysical properties of the water and nanoparticles are given in Table 1 (see Bachok et al. [22] and Turkyilmazoglu [23]). Assuming that the nanofluid is viscous and incompressible and using the nanofluid model proposed by Tiwari and Das [21], the governing boundary layer equations of mass, momentum, and thermal energy for nanofluids can be written as follows (see Tiwari and Das [21]):

$$
\begin{gathered}
\frac{\partial u}{\partial x}+\frac{\partial v}{\partial y}=0 \\
\rho_{\mathrm{nf}}\left(u \frac{\partial u}{\partial x}+v \frac{\partial u}{\partial y}\right)=\mu_{\mathrm{nf}} \frac{\partial^{2} u}{\partial y^{2}} \\
\left(\rho c_{p}\right)_{\mathrm{nf}}\left(u \frac{\partial T}{\partial x}+v \frac{\partial T}{\partial y}\right)=k_{\mathrm{nf}} \frac{\partial^{2} T}{\partial y^{2}},
\end{gathered}
$$

where $y$ is the coordinate measured in the direction normal to the sheet, $u$ and $v$ are the velocity components along the $x$ - and $y$-axes, respectively, $T$ is the nanofluid temperature, $\rho_{\mathrm{nf}}$ is the effective density of the nanofluid, $\mu_{\mathrm{nf}}$ is the effective dynamic viscosity of nanofluid, $k_{\mathrm{nf}}$ is the thermal conductivity, and $\left(\rho c_{p}\right)$ is the heat capacity of the nanofluid, which are given by (see Vajravelu et al. [24] and Narayana and Sibanda [25])

$$
\begin{gathered}
\rho_{\mathrm{nf}}=(1-\phi) \rho_{f}+\phi \rho_{s}, \quad \mu_{\mathrm{nf}}=\frac{\mu_{f}}{(1-\phi)^{2.5}}, \\
\left(\rho c_{p}\right)_{\mathrm{nf}}=(1-\phi)\left(\rho c_{p}\right)_{f}+\phi\left(\rho c_{p}\right)_{s}, \\
\frac{k_{\mathrm{nf}}}{k_{f}}=\frac{\left(k_{s}+2 k_{f}\right)-2 \phi\left(k_{f}-k_{s}\right)}{\left(k_{s}+2 k_{f}\right)+\phi\left(k_{f}-k_{s}\right)},
\end{gathered}
$$

where $\phi$ is the solid volume fraction of the nanofluid, $\rho_{f}$ is the density of base fluid, $\rho_{s}$ is the density of the nanoparticle, $\mu_{f}$ is the dynamic viscosity of the base fluid, $\left(\rho c_{p}\right)_{f}$ is the heat capacity of the base fluid, $\left(\rho c_{p}\right)_{s}$ is the heat capacity of the nanoparticle, $k_{f}$ is the thermal conductivity of the base fluid, and $k_{s}$ is the thermal conductivity of the solid nanoparticle.

Equations (1)-(3) are subjected to the following boundary conditions:

$$
\begin{gathered}
u=u_{w}+A \frac{\partial u}{\partial y}, \quad v=0, \quad T=T_{w} \quad \text { at } y=0, \\
u=0, \quad T=T_{\infty} \quad \text { as } y \longrightarrow \infty
\end{gathered}
$$

where $A$ is the velocity slip factor. It should be mentioned that such slip conditions were recently used in a series of papers [26-31]. It is worth mentioning at this end that fluid flow with slip is important in microelectromechanical systems (MEMS). The flow in these systems deviates significantly from the traditional no-slip flow because of the microscale dimensions of these devices. Rarefied gas flows with slip boundary conditions are often encountered in the microscale devices and low-pressure situations [32].

Following Ishak [33], we introduce the following similarity transformation:

$$
\begin{gathered}
u=c x f^{\prime}(\eta), \quad v=-\left(c v_{f}\right)^{1 / 2} f(\eta), \\
\theta(\eta)=\frac{\left(T-T_{\infty}\right)}{T_{w}-T_{\infty}}, \quad \eta=y \sqrt{\frac{c}{v_{f}}},
\end{gathered}
$$


TABLE 1: Thermo-physical properties of water and nanoparticles.

\begin{tabular}{lccc}
\hline & $\begin{array}{c}\rho \\
\left(\mathrm{kg} \mathrm{m}^{-3}\right)\end{array}$ & $\begin{array}{c}c_{p} \\
\left(\mathrm{~J} \mathrm{Kg}^{-1} \mathrm{~K}^{-1}\right)\end{array}$ & $\begin{array}{c}k \\
\left(\mathrm{~W} \mathrm{~m}^{-1} \mathrm{~K}^{-1}\right)\end{array}$ \\
\hline Pure water $\left(\mathrm{H}_{2} \mathrm{O}\right)$ & 997.1 & 4179 & 0.6130 \\
Copper $(\mathrm{Cu})$ & 8933 & 385.0 & 401.00 \\
Copper oxide $(\mathrm{CuO})$ & 6320 & 531.8 & 76.500 \\
Alumina $\left(\mathrm{Al}_{2} \mathrm{O}_{3}\right)$ & 3970 & 765.0 & 40.000 \\
Titanium oxide $\left(\mathrm{TiO}_{2}\right)$ & 4250 & 686.2 & 8.9538 \\
\hline
\end{tabular}

where primes denote differentiation with respect to $\eta$. Using transformation (6), (1) is automatically satisfied, while (2) and (3), respectively, reduce to the following nonlinear ordinary differential equations:

$$
\begin{gathered}
\frac{\mu_{\mathrm{nf}}}{\mu_{f}} f^{\prime \prime \prime}+\left((1-\phi)+\phi \frac{\rho_{s}}{\rho_{f}}\right)\left(f f^{\prime \prime}-f^{\prime 2}\right)=0, \\
\frac{1}{\operatorname{Pr}} \frac{k_{\mathrm{nf}}}{k_{f}} \theta^{\prime \prime}+\left((1-\phi)+\phi \frac{\left(\rho c_{p}\right)_{s}}{\left(\rho c_{p}\right)_{f}}\right) f \theta^{\prime}=0
\end{gathered}
$$

subject to the boundary conditions

$$
\begin{gathered}
f(0)=0, \quad f^{\prime}(0)=1+\gamma f^{\prime \prime}(0), \quad \theta(0)=1, \\
f^{\prime}(\eta) \longrightarrow 0, \quad \theta(\eta) \longrightarrow 0 \quad \text { as } \eta \longrightarrow \infty,
\end{gathered}
$$

where $\gamma=A \sqrt{c / \nu_{f}}$ is the velocity slip parameter and $\operatorname{Pr}=$ $\mu_{f} / k_{f}$ is the Prandtl number.

Physical quantities of interest are the skin friction coefficient $C_{f}$ and the local Nusselt number $\mathrm{Nu}$, which are defined as

$$
C_{f}=\frac{\tau_{w}}{\rho_{f} u_{w}^{2}}, \quad \mathrm{Nu}=\frac{q_{w}}{k_{f}\left(T_{w}-T_{\infty}\right)},
$$

where $\tau_{w}$ is the surface shear stress and $q_{w}$ is the surface heat flux, which are given by

$$
\tau_{w}=\mu_{\mathrm{nf}}\left(\frac{\partial u}{\partial y}\right)_{y=0}, \quad q_{w}=-k_{\mathrm{nf}}\left(\frac{\partial T}{\partial y}\right)_{y=0} .
$$

Using the similarity variables (6), we obtain

$$
\begin{gathered}
\operatorname{Re}_{x}^{1 / 2} C_{f}=\frac{1}{(1-\phi)^{2.5}} f^{\prime \prime}(0), \\
\operatorname{Re}_{x}^{-1 / 2} \mathrm{Nu}=-\frac{k_{\mathrm{nf}}}{k_{f}} \theta^{\prime}(0),
\end{gathered}
$$

where $\operatorname{Re}_{x}=u_{w} x / v_{f}$ is the local Reynolds number.

It should be mentioned that for a regular fluid $(\phi=0)$ and without $\operatorname{sip}(\gamma=0),(7)$ reduces to

$$
\begin{gathered}
f^{\prime \prime \prime}+f f^{\prime \prime}-f^{\prime 2}=0, \\
\frac{1}{\operatorname{Pr}} \theta^{\prime \prime}+f \theta^{\prime}=0
\end{gathered}
$$

TABLE 2: The values of $\left|f^{\prime \prime}(0)\right|$ and $-\theta^{\prime}(0)$ for $\mathrm{CuO}$-water nanofluid with $\gamma=\phi=0.1$ and $\operatorname{Pr}=6.2$.

\begin{tabular}{lcc}
\hline Step size $h$ & $\left|f^{\prime \prime}(0)\right|$ & $-\theta^{\prime}(0)$ \\
\hline 0.5 & 0.758528 & 1.230633 \\
0.1 & 0.896314 & 1.396718 \\
0.05 & 0.916250 & 1.402676 \\
0.01 & 0.931318 & 1.403155 \\
0.005 & 0.932160 & 1.402452 \\
0.004 & 0.931852 & 1.402065 \\
\hline
\end{tabular}

along with the boundary conditions

$$
\begin{gathered}
f(0)=0, \quad f^{\prime}(0)=1, \quad \theta(0)=1, \\
f^{\prime}(\eta) \longrightarrow 0, \quad \theta(\eta) \longrightarrow 0 \quad \text { as } \eta \longrightarrow \infty .
\end{gathered}
$$

Equation (12) subject to the associated boundary conditions (14) admits the closed-form analytical solution

$$
f(\eta)=1-e^{-\eta}
$$

which has been first reported by Crane [1], while the solution for the thermal field in terms of Kummer's functions is given by Grubka and Bobba [3] as

$$
\theta(\eta)=e^{-\operatorname{Pr} \eta} \frac{M\left(\operatorname{Pr}, \operatorname{Pr}+1,-\operatorname{Pr} e^{-\eta}\right)}{M(\operatorname{Pr}, \operatorname{Pr}+1,-\operatorname{Pr})} .
$$

Thus,

$$
\begin{gathered}
f^{\prime \prime}(0)=-1, \\
\theta^{\prime}(0)=-\operatorname{Pr}+\frac{\operatorname{Pr}^{2}}{\operatorname{Pr}+1} \frac{M(\operatorname{Pr}+1, \operatorname{Pr}+2,-\operatorname{Pr})}{M(\operatorname{Pr}, \operatorname{Pr}+1,-\operatorname{Pr})}
\end{gathered}
$$

where $M(a, b, z)$ denotes the Kummer's function (see Abramowitz and Stegun [34]).

\section{Method of Solution}

The set of ordinary differential equations (7) and (8) are highly nonlinear and cannot be solved analytically. Therefore, the finite element method [35-38] is implemented to solve this system numerically. For computational purposes, $\eta_{\infty}$ has been fixed as 8 . The dimensionless spatial coordinate is discretized by uniform elements. We did experiment with different step sizes $(h=0.5,0.1,0.05,0.01,0.005$, and 0.004$)$ for $\gamma=\phi=0.1$ and $\operatorname{Pr}=6.2$ as shown in Table 2 and observed that a very slight change occurs for step size $h<0.01$, but the computational time increases too much. Thus, for the computational purpose, $h=0.01$ is taken for presentation of the results.

The Gauss quadrature formula has been used to calculate the integrals. Owing to the nonlinearity of the system of equations, an iterative scheme has been used to solve it. An initial guess is taken at each node point. The system of equations is then linearized by incorporating the functions, which are assumed to be known values of the functions $f$ 


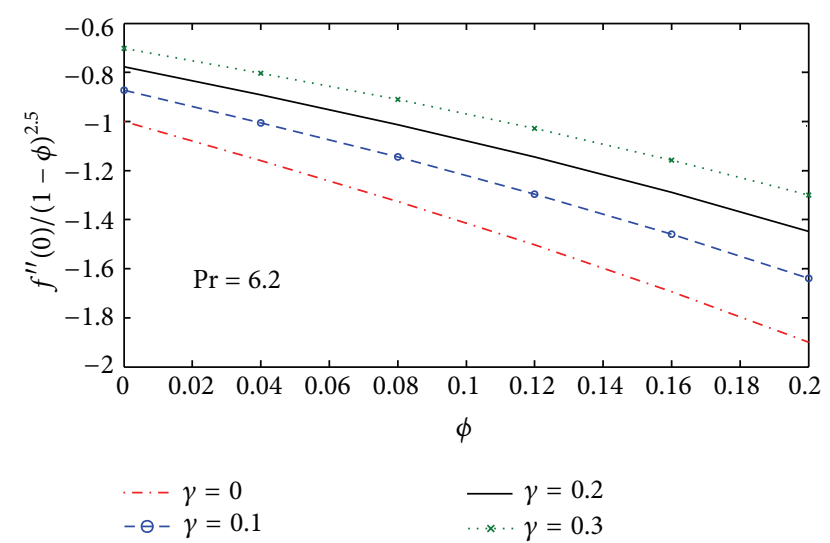

FIGURE 1: Skin friction coefficient against $\phi$ for different values of $\gamma$ for $\mathrm{CuO}$-water nanofluid.

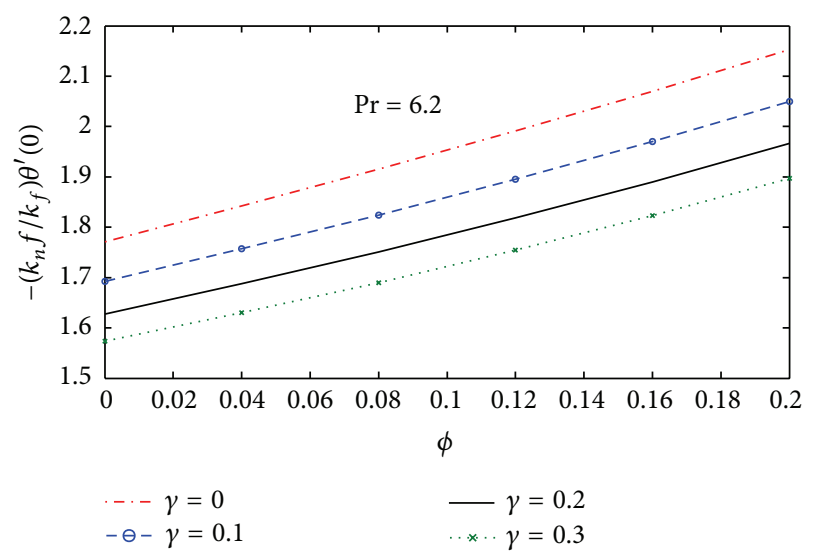

FIGURE 2: Heat transfer coefficient against $\phi$ for different values of $\gamma$ for $\mathrm{CuO}$-water nanofluid.

and $\theta$. After applying the given boundary conditions, the remaining system of equations has been solved using Gausselimination method. This gives us new values of unknowns. This process continues till the absolute differences of two successive iterate values of unknowns are less than the accuracy of 0.0001 .

3.1. Code Verification. In order to verify the accuracy of the applied numerical scheme, comparisons of the present results corresponding to the values of heat transfer coefficient for $\gamma=0$ and $\phi=0$ are made with the available results of Grubka and Bobba [3], Chen [39], Mukhopadhyay et al. [40], and Ishak et al. [41, 42] as well as the series solution given by (17), as presented in Table 3. The results are found in an excellent agreement and thus give confidence that the numerical results in our case are accurate.

\section{Results and Discussion}

Numerical solutions to the system of ordinary differential equations (7) and (8) were obtained using finite element method (FEM). A study has been made on nondimensional parameters and results are presented in Figures 1-10. For

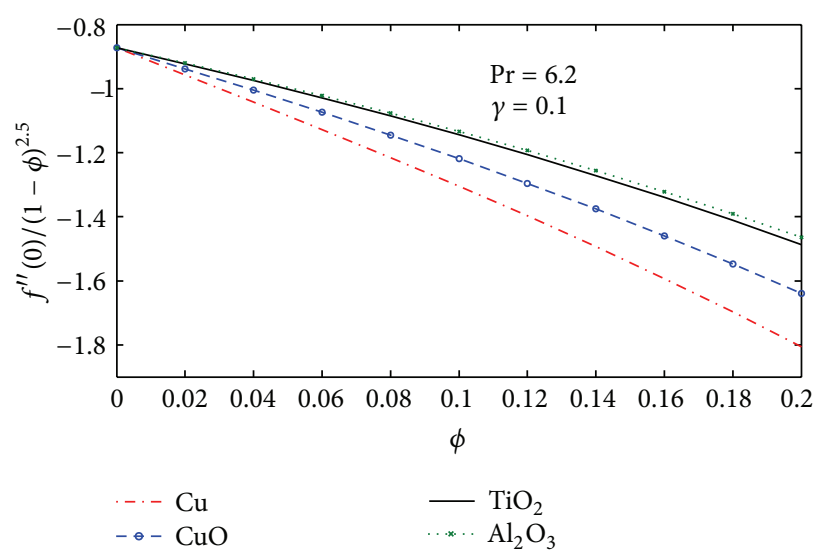

FIGURE 3: Skin friction coefficient against $\phi$ for different water based nanofluids.

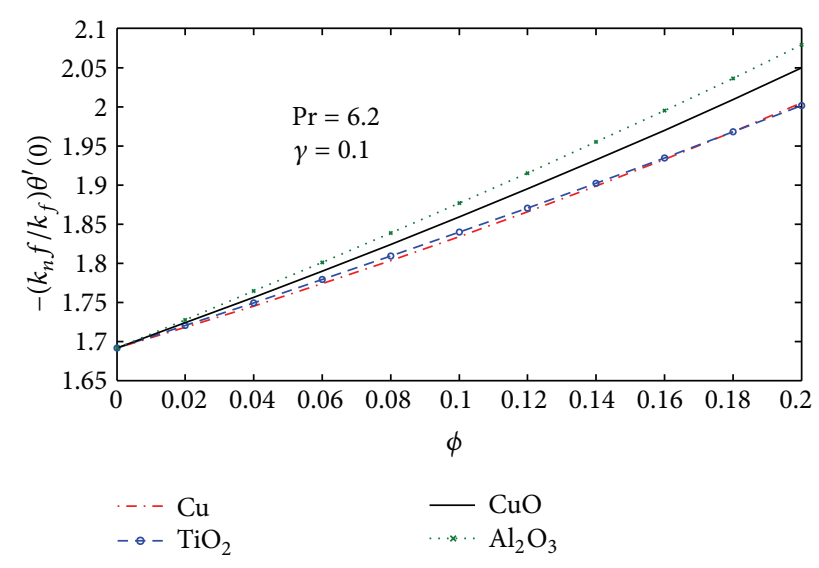

FIGURE 4: Heat transfer coefficient against $\phi$ for different water based nanofluids.

numerical computations, the following default parameter values have been prescribed: $\gamma=\phi=0.1$ and $\operatorname{Pr}=6.2$.

Variations of the reduced skin friction coefficient $f^{\prime \prime}(0) /(1-\phi)^{2.5}$ and the reduced local Nusselt number $-\left(k_{\mathrm{nf}} / k_{f}\right) \theta^{\prime}(0)$ as a function of nanoparticle fraction parameters $\phi$ under different values of the slip parameter $\gamma$ are shown in Figures 1 and 2. It is seen in Figure 1 that the skin friction coefficient increases (in absolute sense) with $\phi$, whereas it decreases with velocity slip parameter. It means that in the no-slip velocity condition $(\gamma=0)$ the highest surface shear stress occurs for pure fluid $(\phi=0)$ as well as nanofluid. These observations (for pure fluid) show good agreement with the results of Mukhopadhyay and Gorla [43].

Figure 2 shows the heat transfer rate with $\phi$ for different values of velocity slip parameter $\gamma$. Heat transfer rate increases with $\phi$ but decreases with velocity slip parameter. Hence, to achieve a high heat transfer rate, less slip on the fluid-solid interface is desired. Also, the heat transfer rate is higher for a nanofluid $(\phi \neq 0)$ compared to a regular fluid $(\phi=0)$. Figures 3 and 4 , respectively, present the variations of the skin friction coefficient and the local Nusselt number with $\phi$ for different types of nanofluids: $\mathrm{Cu}$-water, $\mathrm{TiO}_{2}$-water, $\mathrm{CuO}$-water, and $\mathrm{Al}_{2} \mathrm{O}_{3}$-water. It is seen that the local Nusselt number, which 
TABle 3: Values of $-\theta^{\prime}(0)$ for several values of Prandtl number $\operatorname{Pr}$ with $\gamma=0$.

\begin{tabular}{cccccccc}
\hline$\phi$ & Pr & Reference [3] & Reference [39] & Reference [40] & References [41, 42] & \multicolumn{2}{c}{ Present results } \\
& & & & & & 0.466965907 & 0.4631445610 \\
Analytical equation (17)
\end{tabular}

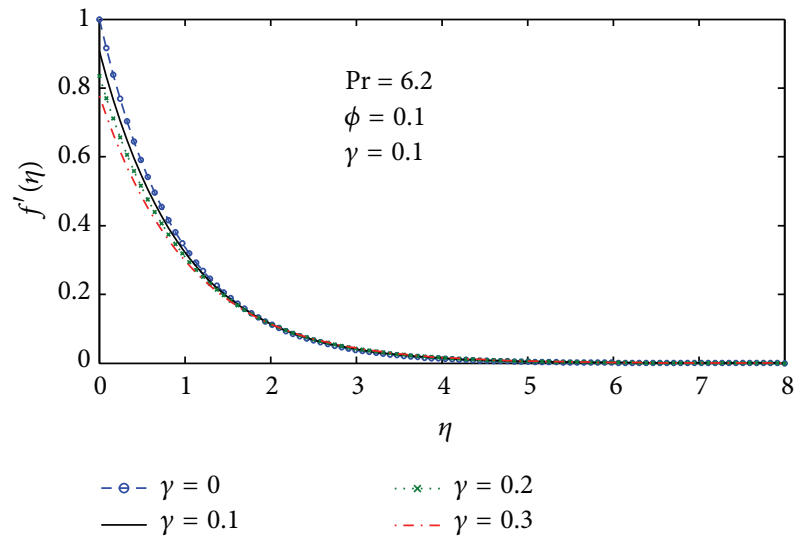

FIGURE 5: Velocity variation with $\eta$ for different values of $\gamma$ for $\mathrm{CuO}$ water nanofluid.

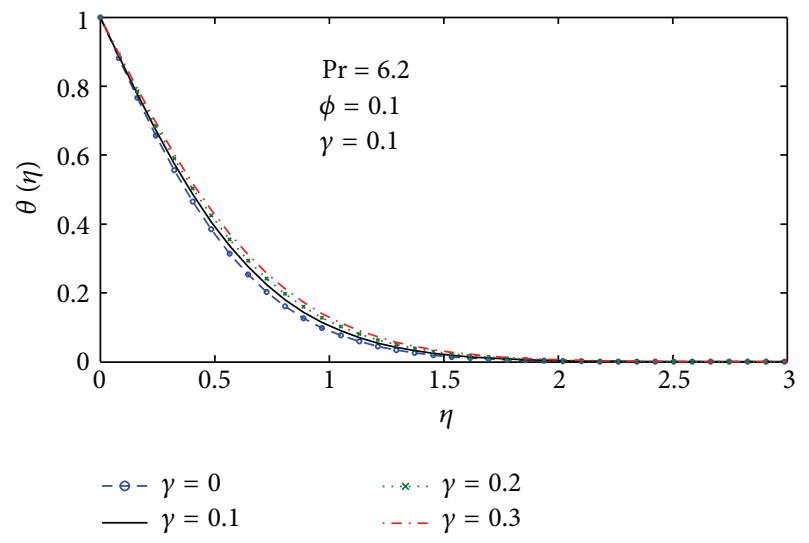

FIGURE 6: Temperature variation with $\eta$ for different values of $\gamma$ for $\mathrm{CuO}$-water nanofluid.

represents the heat transfer rate at the surface, is the highest for $\mathrm{Al}_{2} \mathrm{O}_{3}$-water nanofluid.

The velocity and temperature profiles for different values of the physical parameters are given in Figures 5-10. Figures 5 and 6 show the velocity and temperature distributions with different values of the velocity slip parameter. It is clear from Figure 5 that the velocity decreases in the presence of slip at the fluid-solid interface and decreases monotonically to zero far away from the solid surface. In case of the no-slip condition, the fluid velocity adjacent to the solid surface is equal to the velocity of the stretching sheet; then

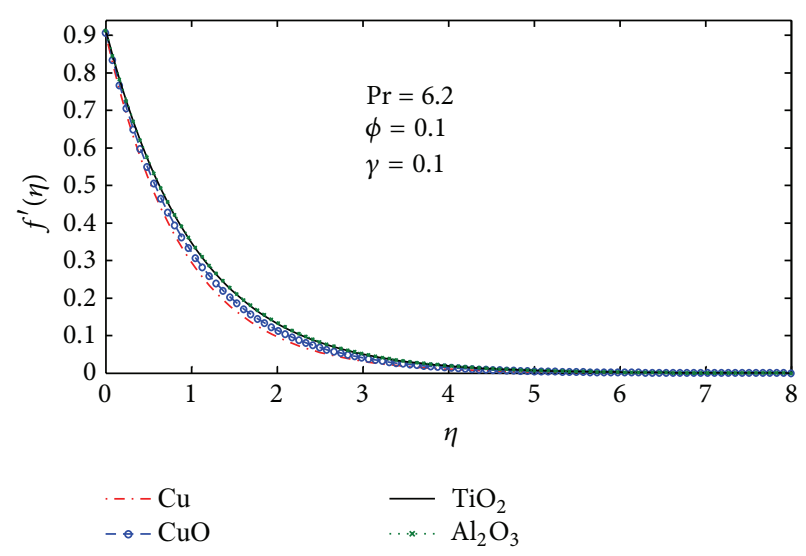

FIGURE 7: Velocity variation with $\eta$ for different water based nanofluids.

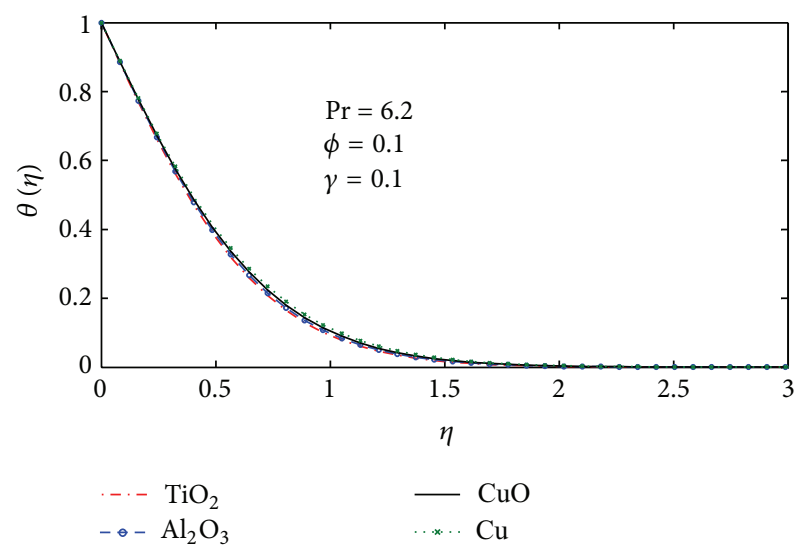

FIgURE 8: Temperature variation with $\eta$ for different water based nanofluids.

$f^{\prime}(0)=1$, which is clearly satisfied in this figure. Figure 6 indicates that an increase in the velocity slip parameter tends to increase the temperature of the fluid and consequently decrease the temperature gradient, which represents the rate of heat transfer. This observation is in agreement with the results presented in Figure 2.

Figures 7 and 8 show the velocity and temperature distributions in the boundary layer for nanofluid with different nanoparticles. It is observed that both velocity and temperature profiles are influenced by the types of the 


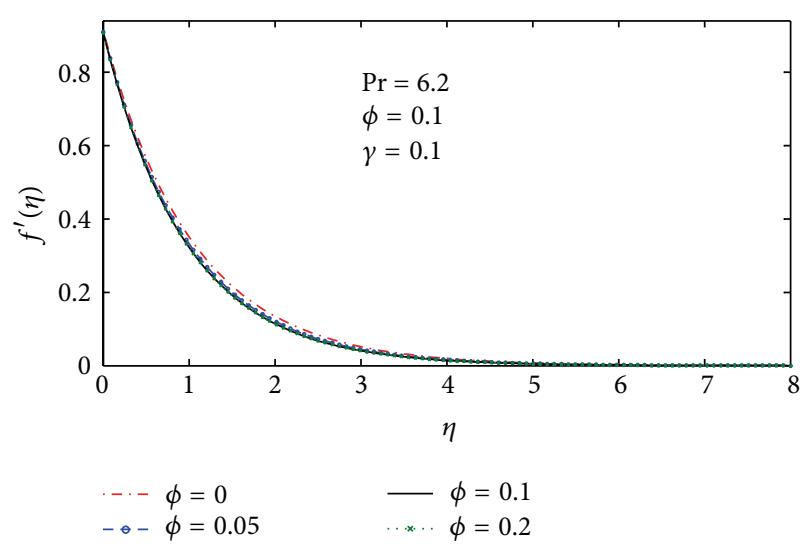

FIGURE 9: Velocity variation with $\eta$ for different values of $\phi$ for $\mathrm{CuO}$ water nanofluid.

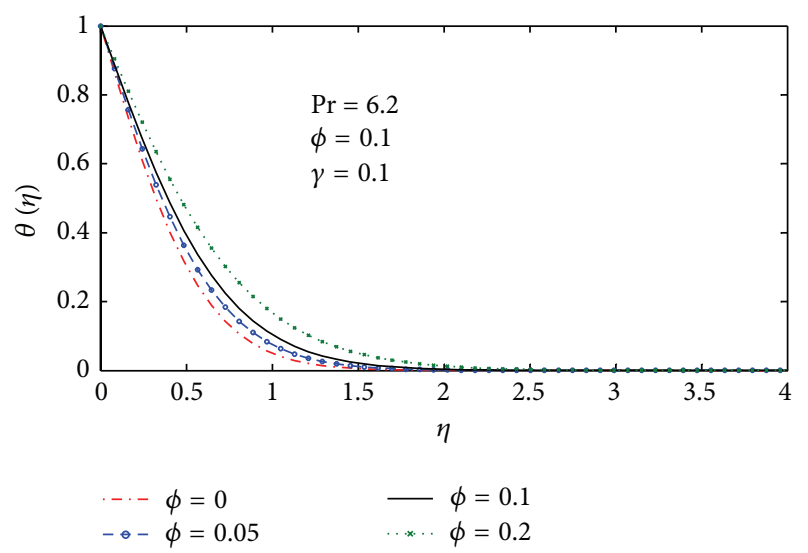

FIGURE 10: Temperature variation with $\eta$ for different values of $\phi$ for $\mathrm{CuO}$-water nanofluid.

nanoparticle. It is also seen that the velocity boundary layer thickness is smaller for $\mathrm{Cu}$ compared to $\mathrm{CuO}, \mathrm{TiO}_{2}$, and $\mathrm{Al}_{2} \mathrm{O}_{3}$ nanoparticles.

Finally, Figures 9 and 10, respectively, depict the effects of nanoparticle volume fraction $\phi$ on the velocity and temperature distributions for $\mathrm{CuO}$-water nanofluid. The effect of $\phi$ on the flow field is less significant than the thermal field. With increasing the concentration of the nanoparticle, the velocity decreases throughout the boundary layer region. It is due to the fact that nanoparticles produce friction in fluid, which retards the flow. Nanoparticles also serve to increase the thermal conductivity of the base fluid; as a result, heat is transferred from the sheet to the fluid with faster rate and consequently warms the thermal boundary layer region. Therefore, as the concentration of the nanoparticles in the base fluid increases, the temperature in the thermal boundary layer also increases, which is clearly visible in Figure 10.

\section{Conclusions}

In this paper, the problem of two-dimensional flow of a viscous and incompressible water based nanofluid over a stretching flat sheet with velocity slip conditions was numerically studied. The governing partial differential equations for mass, momentum, and energy were transformed into ordinary differential equations using a similarity transformation. These equations were solved numerically using finite element method. We found that velocity slip parameter reduces the rate of heat transfer. The results also indicate that with the increase of nanoparticle volume fraction, the skin friction coefficient as well as the heat transfer rate increases.

\section{Acknowledgments}

The authors would like to thank the anonymous reviewers for their constructive comments and suggestions. This work was supported by research Grants from the Ministry of Higher Education, Malaysia (Project Code: FRGS/1/2012/SG04/ UKM/01/1) and the Universiti Kebangsaan Malaysia (Project Code: DIP-2012-31).

\section{References}

[1] L. J. Crane, "Flow past a stretching plate," Für Angewandte. Mathematik Und Physik, vol. 21, pp. 645-647, 1970.

[2] P. S. Gupta and A. S. Gupta, "Heat and mass transfer on a stretching sheet with suction or blowing," The Canadian Journal of Chemical Engineering, vol. 55, pp. 744-746, 1977.

[3] L. J. Grubka and K. M. Bobba, "Heat transfer characteristics of a continuous, stretching surface with variable temperature," Journal of Heat Transfer, vol. 107, no. 1, pp. 248-250, 1985.

[4] H. I. Andersson, J. B. Aarseth, and B. S. Dandapat, "Heat transfer in a liquid film on an unsteady stretching surface," International Journal of Heat and Mass Transfer, vol. 43, no. 1, pp. 69-74, 2000.

[5] A. Ali and A. Mehmood, "Homotopy analysis of unsteady boundary layer flow adjacent to permeable stretching surface in a porous medium," Communications in Nonlinear Science and Numerical Simulation, vol. 13, no. 2, pp. 340-349, 2008.

[6] A. Ishak, R. Nazar, and I. Pop, "Unsteady mixed convection boundary layer flow due to a stretching vertical surface," The Arabian Journal for Science and Engineering B, vol. 31, no. 2, pp. 165-182, 2006.

[7] A. Ishak, R. Nazar, and I. Pop, "Heat transfer over an unsteady stretching permeable surface with prescribed wall temperature," Nonlinear Analysis. Real World Applications, vol. 10, no. 5, pp. 2909-2913, 2009.

[8] A. Ishak, R. Nazar, and I. Pop, "Boundary layer flow and heat transfer over an unsteady stretching vertical surface," Meccanica, vol. 44, no. 4, pp. 369-375, 2009.

[9] T. Hayat, Z. Abbas, and T. Javed, "Mixed convection flow of a micropolar fluid over a non-linearly stretching sheet," Physics Letters A, vol. 372, no. 5, pp. 637-647, 2008.

[10] R. Sharma, "Effect of viscous dissipation and heat source on unsteady boundary layer flow and heat transfer past a stretching surface embedded in a porous medium using element free Galerkin method," Applied Mathematics and Computation, vol. 219, no. 3, pp. 976-987, 2012.

[11] R. Sharma, R. Bhargava, and I. V. Singh, "A numerical solution of MHD convection heat transfer over an unsteady stretching surface embedded in a porous medium using element free Galerkin method," International Journal of Applied Mathematics and Mechanics, vol. 8, no. 10, pp. 83-103, 2012. 
[12] E. M. Sparrow and J. P. Abraham, "Universal solutions for the streamwise variation of the temperature of a moving sheet in the presence of a moving fluid," International Journal of Heat and Mass Transfer, vol. 48, no. 15, pp. 3047-3056, 2005.

[13] S. U. S. Choi and J. A. Eastman, "Enhancing thermal conductivity of fluids with nanoparticles," in Proceedings of the ASME International Mechanical Engineering Congress and Exposition, pp. 12-17, San Francisco, Calif, USA, November 1995.

[14] K. Khanafer, K. Vafai, and M. Lightstone, "Buoyancy-driven heat transfer enhancement in a two-dimensional enclosure utilizing nanofluids," International Journal of Heat and Mass Transfer, vol. 46, no. 19, pp. 3639-3653, 2003.

[15] K. Khanafer and K. Vafai, "A critical synthesis of thermophysical characteristics of nanofluids," International Journal of Heat and Mass Transfer, vol. 54, no. 19-20, pp. 4410-4428, 2011.

[16] K. V. Wong and O. D. Leon, "Applications of nanofluids: current and future," Advances in Mechanical Engineering, vol. 2010, Article ID 519659, 11 pages, 2010.

[17] R. Saidur, K. Y. Leong, and H. A. Mohammad, "A review on applications and challenges of nanofluids," Renewable and Sustainable Energy Reviews, vol. 15, no. 3, pp. 1646-1668, 2011.

[18] D. Wen, G. Lin, S. Vafaei, and K. Zhang, "Review of nanofluids for heat transfer applications," Particuology, vol. 7, no. 2, pp. 141150, 2009.

[19] O. Mahian, A. Kianifar, S. A. Kalogirou, I. Pop, and S. Wongwises, "A review of the applications of nanofluids in solar energy," International Journal of Heat and Mass Transfer, vol. 57, pp. 582-594, 2013.

[20] Y. Ding, H. Chen, L. Wang et al., "Heat transfer intensification using nanofluids," Kona, no. 25, pp. 23-38, 2007.

[21] R. K. Tiwari and M. K. Das, "Heat transfer augmentation in a two-sided lid-driven differentially heated square cavity utilizing nanofluids," International Journal of Heat and Mass Transfer, vol. 50, no. 9-10, pp. 2002-2018, 2007.

[22] N. Bachok, A. Ishak, and I. Pop, "Boundary layer stagnationpoint flow and heat transfer over an exponentially stretching/shrinking sheet in a nanofluid," International Journal of Heat and Mass Transfer, vol. 55, pp. 8122-8128, 2012.

[23] M. Turkyilmazoglu, "Exact analytical solutions for heat and mass transfer of MHD slip flow in nanofluids," Chemical Engineering Science, vol. 84, pp. 182-187, 2012.

[24] K. Vajravelu, K. V. Prasad, J. Lee, C. Lee, I. Pop, and R. A. Van Gorder, "Convective heat transfer in the flow of viscous Agwater and $\mathrm{Cu}$-water nanofluids over a stretching surface," International Journal of Thermal Sciences, vol. 50, no. 5, pp. 843-851, 2011.

[25] M. Narayana and P. Sibanda, "Laminar flow of a nanoliquid film over an unsteady stretching sheet," International Journal of Heat and Mass Transfer, vol. 55, pp. 7552-7560, 2012.

[26] H. I. Andersson, "Slip flow past a stretching surface," Acta Mechanica, vol. 158, no. 1-2, pp. 121-125, 2002.

[27] C. Y. Wang, "Analysis of viscous flow due to a stretching sheet with surface slip and suction," Nonlinear Analysis. Real World Applications, vol. 10, no. 1, pp. 375-380, 2009.

[28] T. Fang, J. Zhang, and S. Yao, "Slip MHD viscous flow over a stretching sheet-an exact solution," Communications in Nonlinear Science and Numerical Simulation, vol. 14, pp. 3731-3737, 2009.

[29] S. Mukhopadhyay and H. I. Andersson, "Effects of slip and heat transfer analysis of flow over an unsteady stretching surface," Heat and Mass Transfer, vol. 45, no. 11, pp. 1447-1452, 2009.
[30] K. Bhattacharyya, S. Mukhopadhyay, and G. C. Layek, "Slip effects on boundary layer stagnation-point flow and heat transfer towards a shrinking sheet," International Journal of Heat and Mass Transfer, vol. 54, no. 1-3, pp. 308-313, 2011.

[31] F. Aman, A. Ishak, and I. Pop, "Mixed convection boundary layer flow near stagnation-point on vertical surface with slip," Applied Mathematics and Mechanics. English Edition, vol. 32, no. 12, pp. 1599-1606, 2011.

[32] T. Fang, S. Yao, J. Zhang, and A. Aziz, "Viscous flow over a shrinking sheet with a second order slip flow model," Communications in Nonlinear Science and Numerical Simulation, vol. 15, no. 7, pp. 1831-1842, 2010.

[33] A. Ishak, "Thermal boundary layer flow over a stretching sheet in a micropolar fluid with radiation effect," Meccanica, vol. 45, no. 3, pp. 367-373, 2010.

[34] M. Abramowitz and I. A. Stegun, Handbook of Mathematical Functions, Dover, New York, NY, USA, 1965.

[35] J. N. Reddy, An Introduction To the Finite Element Method, McGraw-Hill, New York, NY, USA, 1984.

[36] R. Bhargava, R. Sharma, and O. A. Beg, "Oscillatory chemicallyreacting MHD free convection heat and mass transfer in a porous medium with Soret and Dufour effects: finite element modeling," International Journal of Applied Mathematics and Mechanics, vol. 5, no. 6, pp. 15-37, 2009.

[37] R. Sharma, R. Bhargava, and P. Bhargava, "A numerical solution of unsteady MHD convection heat and mass transfer past a semi-infinite vertical porous moving plate using element free Galerkin method," Computational Materials Science, vol. 48, no. 3, pp. 537-543, 2010.

[38] R. Sharma, R. Bhargava, and I. V. Singh, "Combined effect of magnetic field and heat absorption on unsteady free convection and heat transfer flow in a micropolar fluid past a semiinfinite moving plate with viscous dissipation using element free Galerkin method," Applied Mathematics and Computation, vol. 217, no. 1, pp. 308-321, 2010.

[39] C. H. Chen, "Laminar mixed convection adjacent to vertical, continuously stretching sheets," Heat and Mass Transfer, vol. 33, no. 5-6, pp. 471-476, 1998.

[40] S. Mukhopadhyay, I. C. Mondal, and R. S. R. Gorla, "Effects of thermal stratification on flow and heat transfer past a porous vertical stretching surface," Heat and Mass Transfer, vol. 48, pp. 915-921, 2012.

[41] A. Ishak, R. Nazar, and I. Pop, "Mixed convection on the stagnation point flow toward a vertical, continuously stretching sheet," Journal of Heat Transfer, vol. 129, no. 8, pp. 1087-1090, 2007.

[42] A. Ishak, R. Nazar, and I. Pop, "Hydromagnetic flow and heat transfer adjacent to a stretching vertical sheet," Heat and Mass Transfer, vol. 44, no. 8, pp. 921-927, 2008.

[43] S. Mukhopadhyay and R. S. R. Gorla, "Effects of partial slip on boundary layer flow past a permeable exponential stretching sheet in presence of thermal radiation," Heat and Mass Transfer, vol. 48, pp. 1773-1781, 2012. 


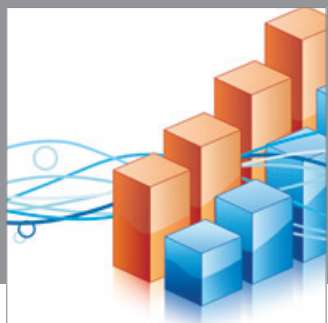

Advances in

Operations Research

mansans

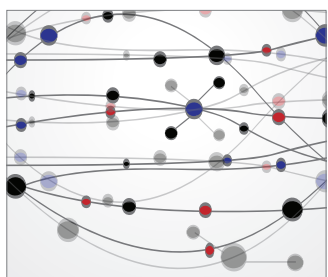

The Scientific World Journal
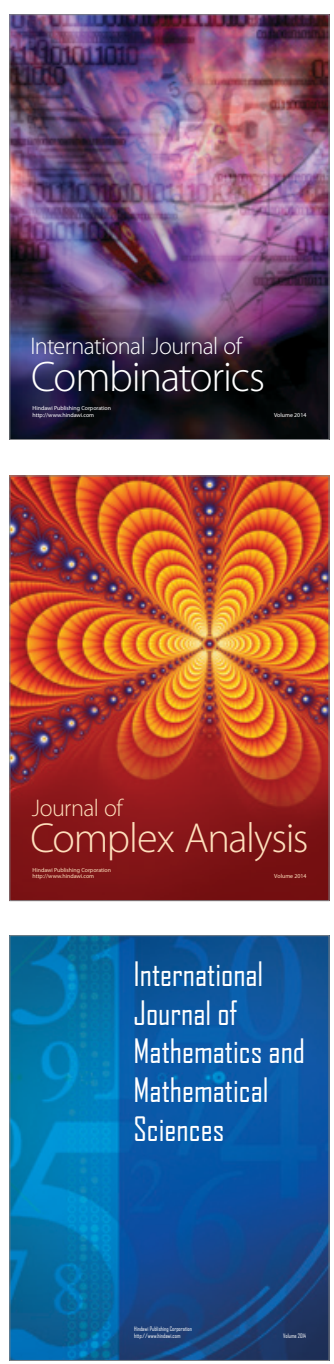
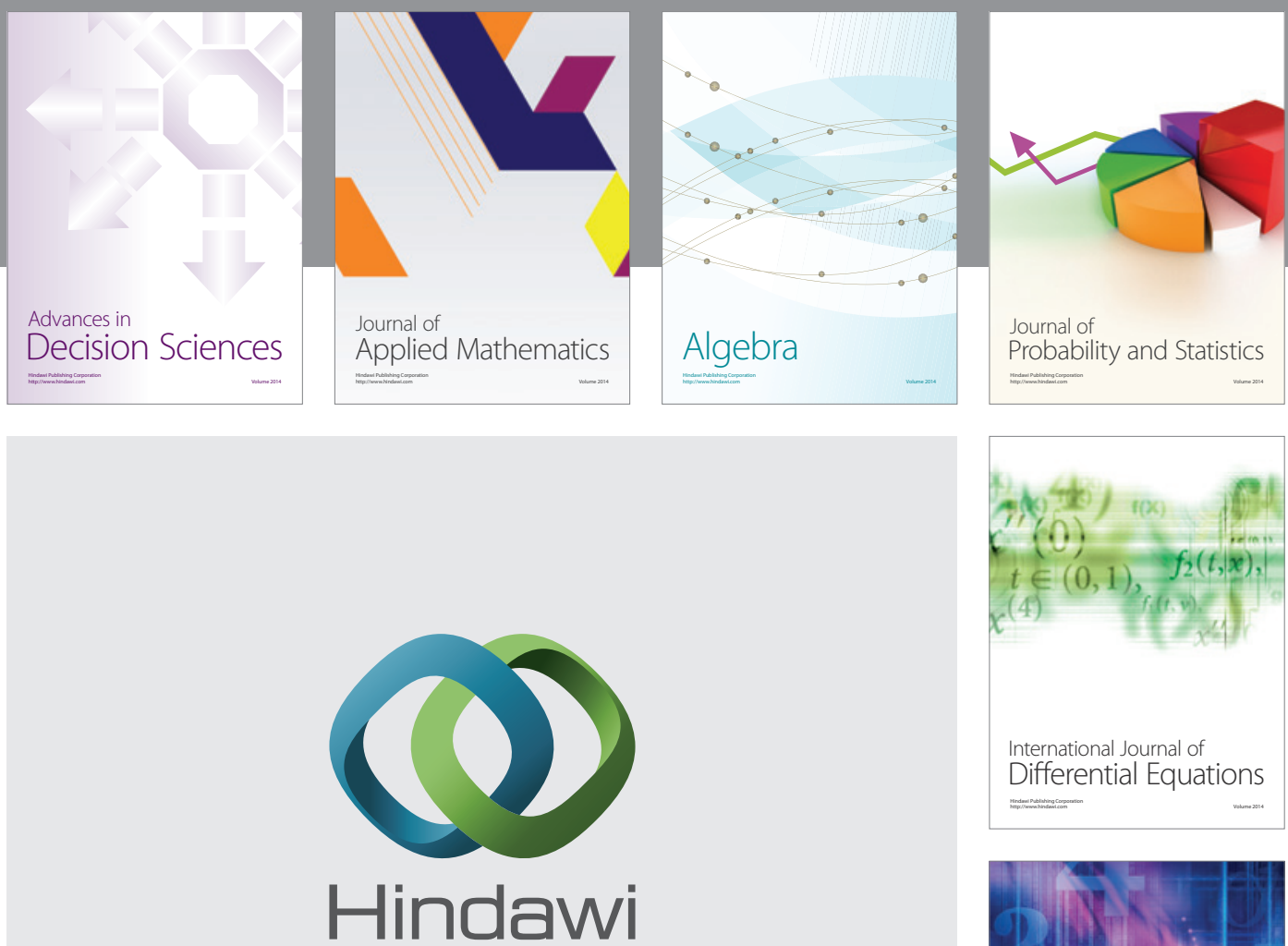

Submit your manuscripts at http://www.hindawi.com
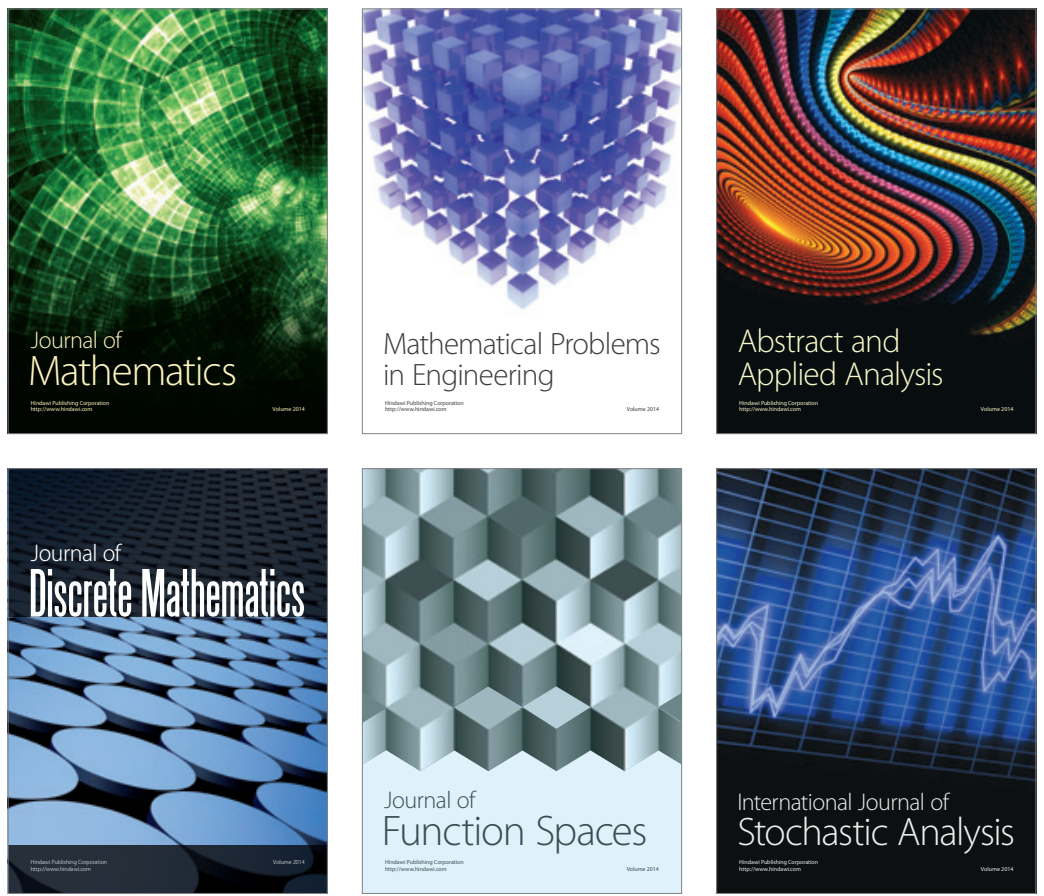

Journal of

Function Spaces

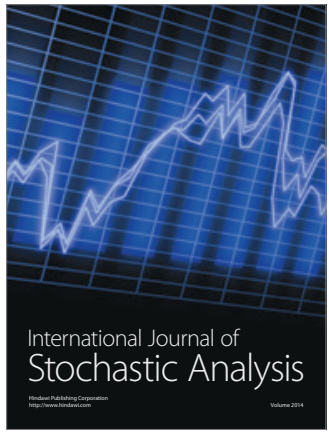

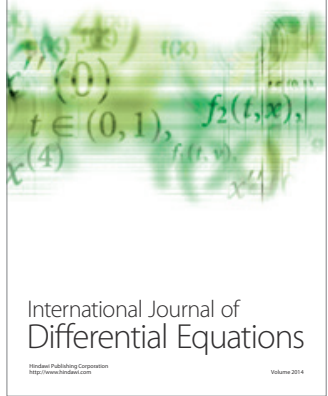
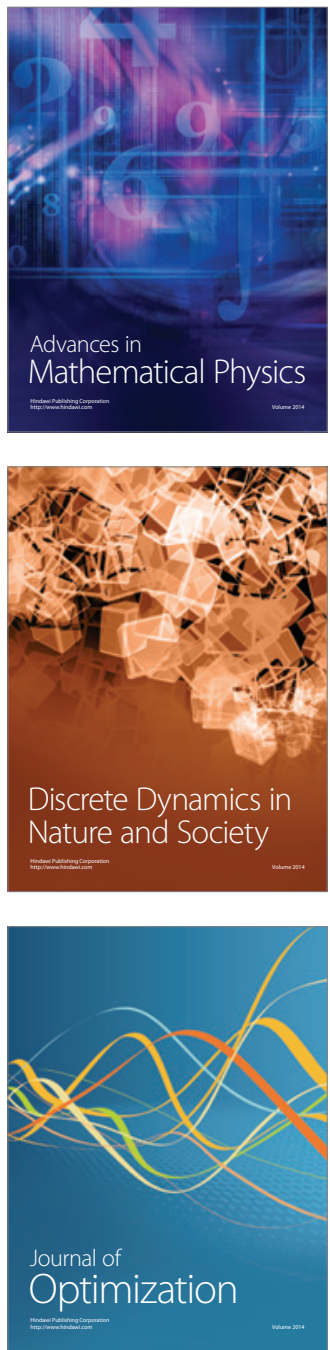